Bull. Mater. Sci., Vol. 22, No. 5, August 1999, pp. 829-834. Indian Academy of Sciences.

\title{
Computer modelling and simulation in materials science
}

\author{
N RAMAKRISHNAN* and P RAMARAO ${ }^{\dagger}$ \\ Computer Simulation Centre, Defence Metallurgical Research Laboratory, Kanchanbagh, Hyderabad 500 058, India \\ ${ }^{\dagger}$ Jawaharlal Nehru Centre for Advanced Scientific Research, Jakkur P.O., Bangalore 560 064, India

\begin{abstract}
The paper presents a brief overview of different types of modelling and simulation along with the distinguishing features between the two. Spatial as well as temporal size scales with a special reference to multiscale modelling are explained with illustrations. The paper includes a discussion on numerical experiments and their validation based on the authors' work on FEM simulation of crack-tip blunting during ductile fracture. Attention is drawn to the use of a $3 P$ technique involving integrated simulation of deformation (property) at microstructural level, the process at macroscopic level and the performance at the product level.
\end{abstract}

Keywords. Computer modelling; computer simulation; materials science; numerical experiment; size scales.

\section{Introduction}

Computer modelling and simulation are known to aid design of new materials, processes and products. In this context, we shall present a brief discussion on certain generic concepts that have provided the base for the investigations involving modelling or simulation in materials science. We will show how a properly validated numerical simulation can substitute an actual experiment in generating reliable data, using our work on crack-tip blunting during ductile fracture.

\section{Modelling}

A model is a conceptual description of an entity or a phenomenon, quantified using the relevant laws of science with allowable approximations and simplifications. The four important sequential steps involved in any modelling are conceptualization, simplification, representation and finally quantification.

We model a porous material as a hollow sphere, fibre composite as a concentrically placed cylinder, an atom as a rigid sphere, a statistically homogeneous structure as a continuum and so on. The above examples correspond to the geometry of a system we are interested in. Similarly, modelling can be resorted to for representing material behaviour such as power-law for material flow or exponential law for thermal effects. Modelling involves imposing boundary conditions to describe the interaction of an isolated system with its surroundings in spatial scale and initial conditions to represent the initial configuration of the system in temporal scale.

\footnotetext{
*Author for correspondence
}

Analytical modelling using differential or statistical equations provides a solution in an explicit quantitative form. When such a model is mathematically intractable we resort to numerical modelling. When the basic understanding itself eludes us we make a compromise and rely on empirical modelling. Certain dimensionless parameters provide an ideal source for semi-empirical modelling. For example, a high strength material may be replaced by plasticine for a given analysis provided the strength-to-modulus ratios of the two are alike. In some cases, modelling is done invoking geometric similarities such as length-to-diameter ratio. In materials science, models are mostly categorized as phenomenological, mechanistic, heuristic or statistical depending on the method adopted for modelling.

\section{Simulation}

The two words 'modelling' and 'simulation' are used interchangeably in some contexts perhaps because of their blended role. However, the two expressions are not synonymous. Essentially, a model provides the basis for simulation to create an image of a real system or a phenomenon.

Modern computers have made simulation attractive to the extent that 'virtual reality' has become possible. Sound numerical simulations are almost equivalent to performing real experiments and therefore referred to as 'numerical experiments'. A real experiment is a direct dialogue with the system of interest and therefore expected to be naturally reliable. On the other hand, a numerical experiment derives its strength from the underlying physical model and the extent of validation. We will discuss 'validation' in \$ 5.3.

The sequential steps involved in a typical computer simulation are modelling, digitization, numerical 
computation followed by graphical representation. The numerical computation involves solving the differential equation as described by the model. The user-friendly methods such as the finite element method (FEM), the finite difference method (FDM) and the finite volume method (FVM) are basically the adapted versions of the differential equations for numerical computation.

\section{Size scales}

The physical laws that govern material behaviour differ with the size scale of interest. Modelling in atomic scale $\left(10^{-10}\right.$ to $\left.10^{-8} \mathrm{~m}\right)$ employs inter-atomic potentials and forces to study crystal defects, atomic configurations, nano-scale materials and the like. Microscopic scale $\left(10^{-8}\right.$ to $10^{-6} \mathrm{~m}$ ) deals with entities such as dislocations, grain boundaries and interfaces. The behaviour of a group of grains where homogeneity cannot be assumed falls under the category of meso scale $\left(10^{-6}\right.$ to $\left.10^{-3} \mathrm{~m}\right)$. Polycrystalline plasticity is a meso-scale deformation. Larger than meso scale is termed as macro-scale. The continuum approximation is normally allowed in macro-scale since statistical averaging is possible or, in other words, homogeneity can be assumed. Many practical applications in materials processing operations such as forming, solidification, heat transfer are generally treated under continuum approximations.

Normally, simulation pertaining to a particular size scale provides input to the next higher scale. For instance, simulation of powder aggregates is carried out at the meso-scale (Ramakrishnan et al 1984, 1996; Ramakrishnan and Arunachalam 1993) to obtain the densification behaviour and to be used in the simulation of hot isostatic pressing of a aero-engine turbine disc. Similarly, dislocation studies at atomic scale are employed for understanding dislocation dynamics in microscopic scale. The multi-scale models (Campbell 1998) address the issues pertaining to different size scales and the inter-relations between the scales. There are generic models, which allow analysis of meso-scale or micro-scale problems with well established continuum equations, such as the composite sphere model, the self consistent model, the differential model, the Mori-Tanaka model and an overview of these models is presented by Christensen (1990). The use of FEM for meso-scale simulation has been reviewed by Ramakrishnan (1997).

Scales in temporal axis too warrant similar attention. When the rate of loading is very high (strain rate $\sim 10^{4}$ to $10^{6} / \mathrm{sec}$ ), modelling of pressure transmission through a material involves the wave propagation characteristics at microsecond scale. On the other hand, in the case of time dependent creep deformation (strain rate $\sim 10^{-5}$ to $\left.10^{-7} / \mathrm{sec}\right)$, since loading and relaxation occur at a comparable level, modelling is done at 'mega second' $(1000 \mathrm{~h})$ time scale. Atomic vibrations fall in the realm of nano seconds and biological responses are of the order of microseconds. Analogous to continuum approximation, 'time-scale independence' is assumed wherever applicable like Fourier's law governing heat transfer or Hooke's law pertaining to elastic deformation.

\section{Numerical simulation}

Any numerical simulation of an experiment can be considered quite equivalent to performing the experiment itself, provided the supporting fundamental equations are sound and the input data are precise. We briefly present here our recent investigation (Suresh et al 1999) in which we performed a set of numerical simulations to generate the required fracture mechanics data conveniently and speedily.

\subsection{Experiments to determine $J$}

The experimental method suggested by ASTM (1986, 1987) to determine the ductile fracture initiation toughness parameter $J$ is widely practised. Here the critical value of $J\left(J_{\mathrm{IC}}\right)$ is given by the intercept of the crack tip blunting line, the line that represents the apparent crack advance due to crack tip blunting, with the stable R-curve that represents the actual crack propagation.

An alternative procedure for the determination of $J_{\mathrm{IC}}$ is given by JSME (1981). Here, the width of the featureless zone appearing due to blunting at the crack tip, as observed in a scanning electron microscope is measured and the stretch zone width (SZW), so measured, allows determination of $J_{\mathrm{IC}}$ as per the following equation:

$$
J_{\mathrm{IC}}=m \sigma \mathrm{CTOD}_{\mathrm{C}}=m \sigma\left(2 \mathrm{SZW}_{\mathrm{C}}\right)
$$

where $\sigma$ is a stress measure, $\mathrm{CTOD}_{\mathrm{C}}$ denotes the critical crack tip opening displacement and $m$, that affects the slope $(m \sigma)$ of the blunting line referred to above, is regarded as a material constant. ASTM (1986) prescribes the $m$ value to be 1.0 for a stress measure $\sigma_{\mathrm{yt}}$ which is the average of the yield strength $\left(\sigma_{0}\right)$ and the ultimate tensile strength $\left(\sigma_{u}\right)$.

Both the above methods depend on the accuracy of the slope of the blunting line. A few investigators (Kobayashi et al 1977; Landes and Begley 1974; Paranjpe and Banerjee 1979; Mills 1981; Srinivas et al 1994a) have reported their experimentally determined $m$ values, each corresponding to a specific material and a stress measure. These $m$ values differ widely and particularly for higher values of strain hardening exponents the dispersion is significantly high. 


\subsection{Numerical experiment}

With the exception of Shih (1981), the variation of $m$ has not been investigated analytically or numerically. We carried out numerical simulations using a large deformation FEM (Ramakrishnan et al 1999) to determine $J$ and CTOD for a range of normalized yield strength values $\left(\sigma_{0} / E\right)$ and strain hardening exponents $(n)$, and analysed the blunting line slope for different stress measures. The large deformation analysis becomes necessary since the material undergoes a large strain and rotation at the crack tip.

A planar FEM mesh model of ASTM E 813-81 standard $C(T)$ fracture specimen, shown in figure 1 , is used assuming one-half symmetry. Since our goal is to study deformation at the crack tip (meso-scale) as well as load-displacement variation for the entire specimen (macro-scale), the element size is increased in geometric progression from the crack tip to the outer boundary. A magnified view of the initial mesh morphology around the crack tip is shown in figure $2 a$ and the view of the corresponding deformed mesh in figure $2 b$. The nodes falling on the line $\mathrm{AB}$ (figure 1) were arrested in the $\mathrm{Y}$ direction and the load point $C$ in the $\mathrm{X}$-direction. The load is applied in steps of Y-directional displacements at node $C$. The flow behaviour of the material is assumed to follow the power-law

$$
\sigma_{\text {flow }}=K \varepsilon^{n}
$$

where $\sigma_{\text {flow }}$ is the true flow stress, $K$ the strength coefficient, $\varepsilon$ the elastoplastic strain and $n$ the strain hardening exponent. The investigation comprised a

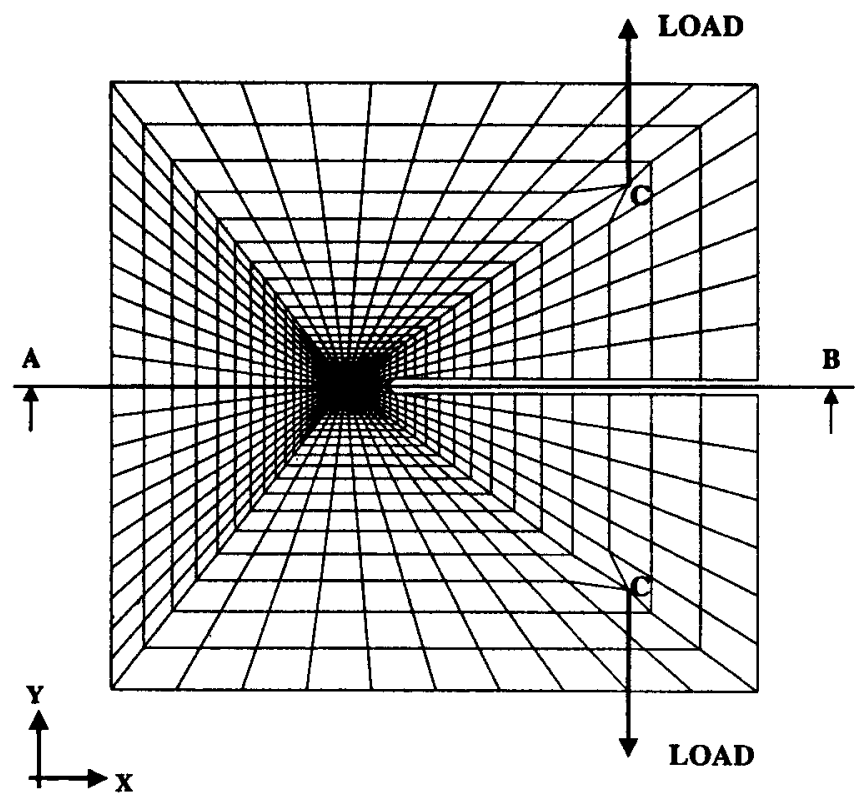

Figure 1. Finite element mesh representation of compact tension specimen. number of FEM simulations of the real experiment. Varying the material properties, the CTOD and the $J$ values were computed as a function of the load-line displacement. The CTOD was measured using $45^{\circ}$ line method as shown in figure $2 \mathrm{~b}$.

\subsection{Validation}

Any modelling or simulation has to be rigorously validated to confirm the correctness of the general constitutive basis as well as to verify the specific applicability to the problem of interest. An ideal validation should take into account the available analytical as well as experimental results.

\section{Initial configuration}

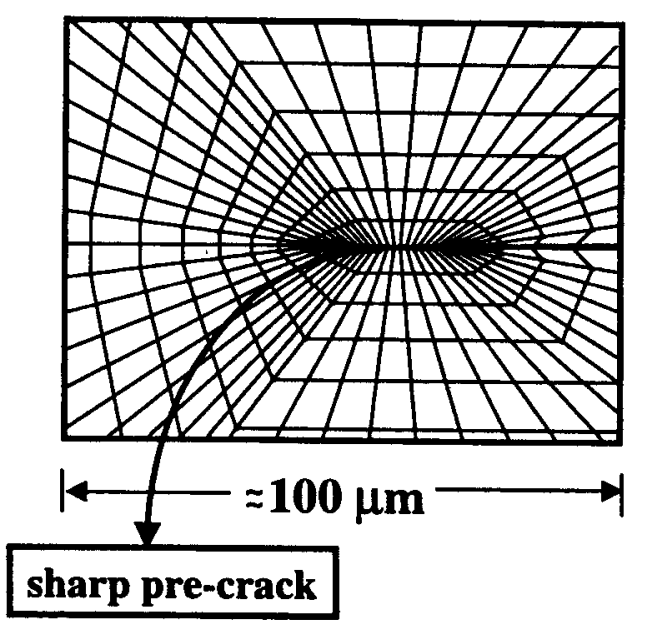

\section{Deformed configuration}

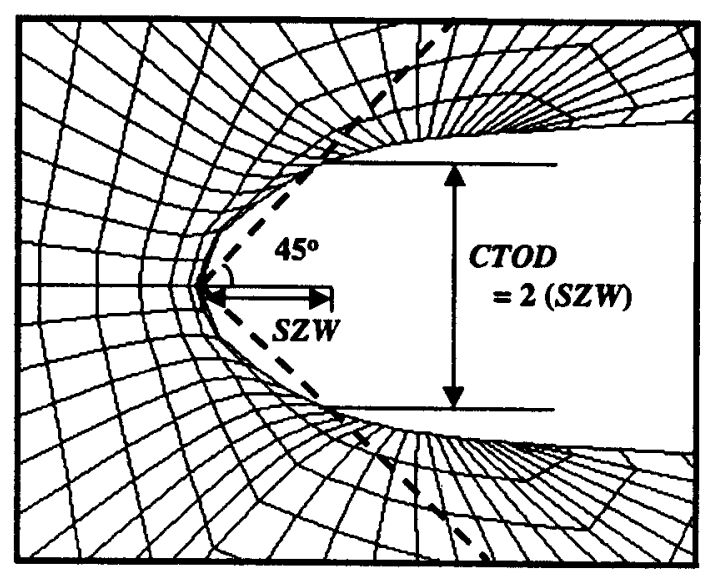

(b)

Figure 2. Finite element mesh morphology at the crack tip (magnified view). 
In the present case, since the analysis pertains to large deformation plasticity, the FEM algorithm was first tested for its accuracy in handling large strain and material rotation (Ramakrishnan et al 1999). A set of available analytical results (Burke and Nix 1979; Okada et al 1990) and the experimental data (Norris et al 1978) on the deformation of a highly ductile tensile specimen were used for the validation. The algorithm thus qualified for its general applicability is then tested for the specific problem of our interest pertaining to fracture specimen with the analytical elastic stress distributions (Meguid 1989) and finally with the experimental data (Srinivas et al 1994b) for $J_{\mathrm{IC}}$ of armco iron.

\subsection{Results and discussion}

In the numerical experiments carried out by us using the suitably validated FEM program, the parameter of interest was $m$ that influences the slope $(m \sigma)$ of the blunting line. The focus was also on the correct choice of the stress measure $\sigma$ in (1). The variation of $m(1.2>m>5)$, as a function of $\sigma_{0} / E$ as well as the strain hardening exponent $(n)$, determined using our numerical experiments (Suresh et al 1999) for the stress measure $\sigma_{0}$ is depicted in figure 3a. The variation of $m$ obtained by Shih (1981), assuming the same stress measure, is presented in figure $3 \mathrm{~b}$. In both the cases the $m$ value is constant in the entire range of $\sigma_{0} / E$ for $n=0.0$ perhaps because the flow stress becomes invariant with strain. For $n>0$, the $m$ value varies widely for $\sigma_{0} / E$ varying from 0.001 to 0.01 in both the cases. However, there is a large difference between the results of our work and those of Shih, particularly for lower $\sigma_{0} / E$ and higher values of $n$. We attribute this mainly to the small scale yielding assumed in Shih's work.

Figure 3c shows the variation of $m$ for the ASTM suggested average stress $\left(\sigma_{y t}\right)$ as the stress measure. Here, the range of $m$ value is reduced to $1.2<m<3.2$. The $m$ values obtained experimentally by Mills (1981) for Incoloy-800, by Paranjpe and Banerjee (1979) for ship building steel, by Srinivas et al (1994a) for cobalt and manganese steels and by Srinivas et al (1994b) for armco iron show a satisfactory agreement with the FEM generated $m$ values within a range of about $10 \%$. The details are given in Suresh et al (1999). When we found the numerical simulation to be reliable and promising, we made an attempt to improve the stress measure to further reduce the range of $m$.

The 'yield strength' $\left(\sigma_{0}\right)$ as a stress measure does not reckon with the behaviour of the material during strain hardening subsequent to yielding. The average stress $\left(\sigma_{y t}\right)$, although takes the hardening into account, implicitly assumes the variation to be linear. To account for the commonly observed non-linearity, we considered the following stress measure based on an integral average:

$$
\sigma=\sigma^{*}=\frac{\int_{0}^{\varepsilon_{0}^{*}} \sigma \mathrm{d} \varepsilon}{\int_{0}^{\varepsilon^{*}} \mathrm{~d} \varepsilon}
$$

The numerator corresponds to the area under the stressstrain curve up to a limiting strain $\left(\varepsilon^{*}\right)$ and represents the toughness of the ductile material. Since the denominator is the limiting strain itself, the division yields an integral average of the true stress $\left(\sigma^{*}\right)$. The average energy approach has been found useful in explaining the mechanical behaviour of materials in a number of practical situations like solid particle erosion (Sundararajan 1993), fatigue failure (Eswara Prasad et al 1996) and these are summarized in a review article by Ramarao (1995).

In the evaluation of $\varepsilon^{*}$, assigning a magnitude for the limiting strain becomes an issue. For the specific case like power-law variation, we assumed the limiting strain to be the strain hardening exponent $(n)$, as Consideré instability sets in beyond this strain in a tensile specimen. This strain corresponds to the beginning of the pronounced void growth and coalescence that precede ductile fracture in the tensile specimen. We used this instability strain as the limit in the integral averaging since $J_{\mathrm{IC}}$ is actually initiation fracture toughness. Accordingly, (3) reduces to,

$$
\sigma^{*}=\frac{K n^{n}}{n+1}
$$

The use of this stress measure markedly reduces the range of $m$ to $1.1<m<1.4$ as shown in figure $3 d$. The average $m$ then turns out to be 1.25 and it is nearly independent of $\sigma_{0} / E$ and $n$. The material-independent character of $m^{*}$ makes it scientifically elegant and practically convenient.

This result throws light on the decade long efforts with regard to the choice of ' $m$ ' and brings out the importance of the correct choice of the stress measure, and thus obviates the discrepancies involved in the determination of $J_{\text {IC }}$ by the experimental methods. Establishing such results by means of real experiments is an arduous task since it involves a variety of materials and a large number of trials not to mention the near impossibility of covering as wide a range of $\sigma_{0} / E$ and $n$ values as has been done in the numerical exercise. Under these circumstances numerical experiments are undoubtedly attractive.

\section{Integrated 3P simulation}

Modelling and simulation are useful in three different areas in developing materials: (i) The micro structures can be modelled using FEM with which the mechanical, electrical, magnetic, physical and thermal properties can be estimated given the properties of the individual phases. 


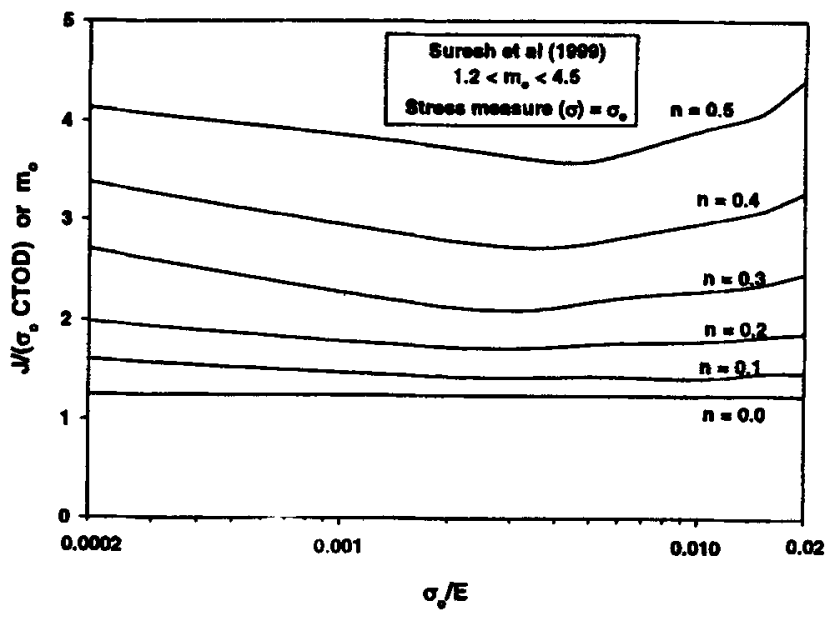

(a)

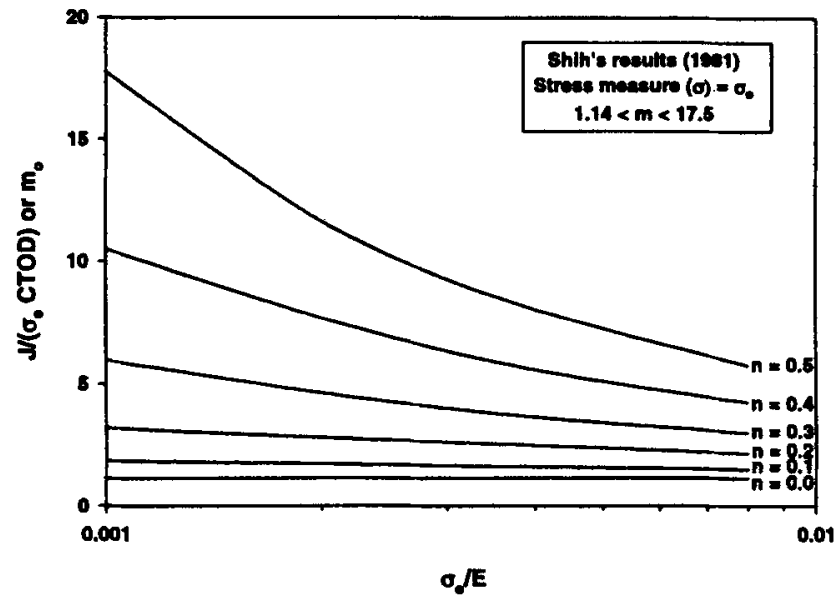

(b)

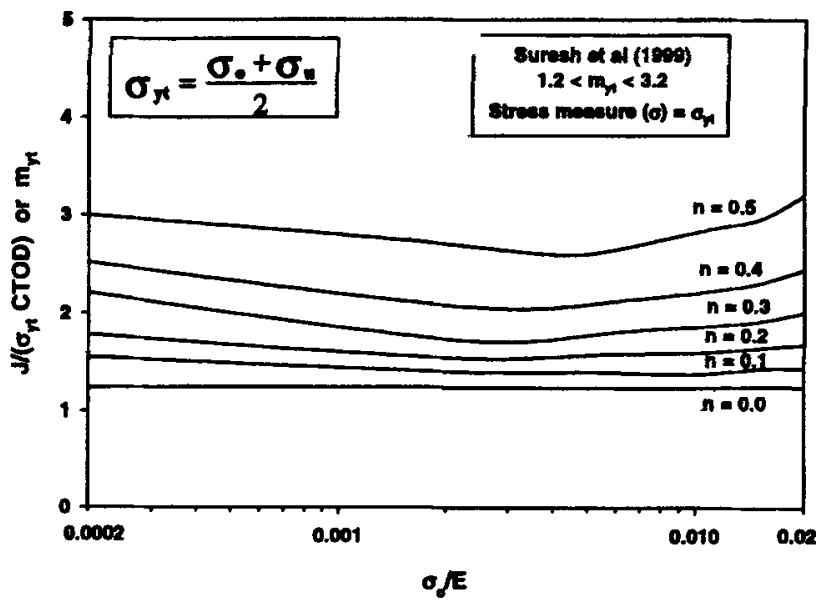

(c)

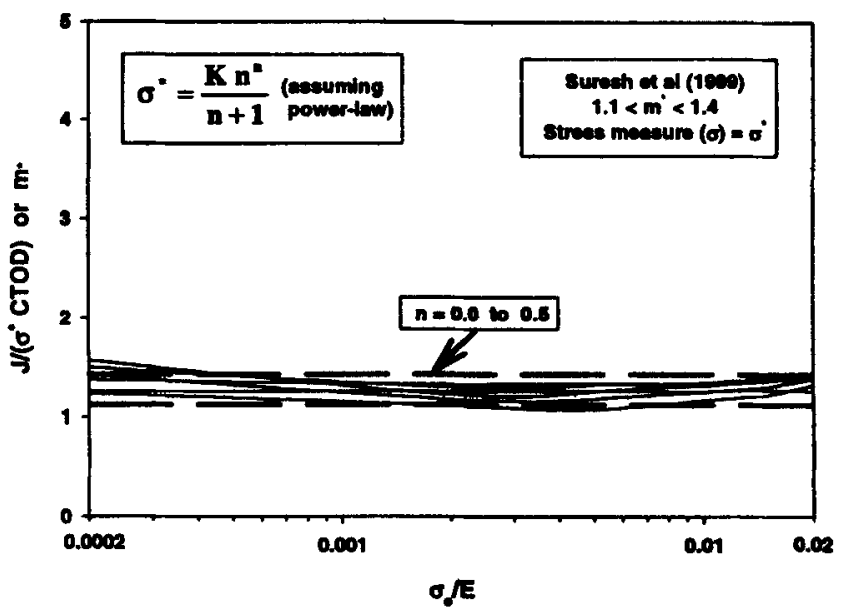

(d)

Figure 3. Slope parameter of the blunting line as a function of normalized yield strength for different strain hardening rates.

These hypothetical microstructures are characterized by the size and shape of the different phases, and in addition by their spatial, size and orientation distributions for different volume fractions of the phases. This method has been found useful in designing metal and ceramic matrix composites (Ramakrishnan 1996; Ramakrishnan et al 1996). (ii) Processes such as casting (Flemings 1998), metal forming (Kobayashi 1982; Rowe et al 1991) and heat treatment (Dowling and Nagendra Palle 1998) can be simulated at macroscopic scale to optimize the process parameters. (iii) Finally, the component subjected to service conditions is simulated to verify the performance of the product. The Defence Metallurgical Research Laboratory at Hyderabad and the Regional Research
Laboratory at Bhopal together have employed an integrated approach for the above three types of simulation termed as 3P simulation (property, process and performance). The application relates to the development of automobile wheel brake drums using a metal matrix composite and the results will be published elsewhere.

The main advantage of the above integrated approach is that it directly links the material selection as well as the process optimization to the performance maximization of the product. That is, the material and the process design take into account the spatial variation of the stresstemperature state in the component and aim at an overall optimization. This is required not only to ensure the expected performance at the critical locations in 
the component but also to avoid unwarranted over design.

The concept of integrated 3P simulation for product development covering property, process and performance is in principle extendable to downstream as well as upstream ends of the 3Ps mentioned above. Beginning with extraction of raw materials from naturally occurring ore bodies, the materials cycle (Cohen 1994) involves several stages of conversion and refining before engineered materials are made and developed into products for society. Once the products have ended their service life, they are either discarded or recycled in a way as to feed back into the stage of product development. The grandest integrated modelling and simulation exercise would be for it to encompass the entire chain of events in the materials cycle not only for purposes of optimizing the processes involved but also to achieve truly sustainable materials development.

\section{Acknowledgements}

The authors thank Prof. T C Rao, Director and Dr S Das, Regional Research Laboratory, Bhopal, for the discussions related to 3P technique. The support of DRDO is acknowledged.

\section{References}

ASTM standard (E 813-8) 1986 Annual Book of ASTM Standards Philadelphia p. 768

ASTM standard (E 1152-87) 1987 Annual Book of ASTM Standards Philadelphia p. 814

Burke M A and Nix W D 1979 Int. J. Solids Struct. 15379

Campbell G H 1998 Mater. Sci. Eng. A251 1

Christensen R M 1990 J. Mech. Phys. Solids 38379

Cohen M 1994 MRS Bull. 193

Dowling W E and Nagendra Palle 1998 ASM HandbookMaterials selection and design 20774
Eswara Prasad N, Malakondiah G, Kutumbarao V V and Rama Rao P 1996 Mater. Sci. Tech. 12563

Flemings M C 1998 Modelling of casting, welding and advanced solidification processes VIII (eds) B G Thomas and C Beckerman (TMS) p. 1

JSME (S001) 1981 Japan Society of Mechanical Engineersstandards

Kobayashi S 1982 J. Appl. Metalworking 2163

Kobayashi H, Hirano K, Nakamura N and Nakazawa H 1977 Proceedings of the 4th int. conf. on fracture (ICF4) 3 p. 583

Landes J D and Begley J A 1974 ASTM STP 560170

Meguid S A (ed.) 1989 Engineering fracture mechanics (London and New York: Elsevier applied science)

Mills W J 1981 J. Testing \& Evaluation 956

Norris Jr D M, Moran B, Scudder J K and Quinones D F 1978 J. Mech. Phys. Solids 261

Okada H, Rajiyah H and Atluri S N 1990 Int. J. Numer. Methods Eng. 2915

Paranjpe S A and Banerjee S 1979 Eng. Fract. Mech. 1143

Ramakrishnan N 1996 Acta Metall. Mater. 421113

Ramakrishnan N 1997 Bull. Mater. Sci. 20885

Ramakrishnan N and Arunachalam V S 1993 J. Am. Ceram. Soc. 762745

Ramakrishnan N, Balakrishna Bhat $\mathrm{T}$ and Arunachalam V S 1984 Acta Metall. 32357

Ramakrishnan N, Arun Kumar M and Radhakrishna Bhat B V 1996 J. Mater. Sci. 313507

Ramakrishnan N, Krishna M Singh, R K V Suresh and Srinivasan N 1999 J. Mater. Process. Technol. 86190

Ramarao P 1995 J. Indian Inst. Sci. 305

Rowe G W, Sturgess C E N, Hartley P and Pillinger I 1991 Finite element plasticity and metal forming analysis (Cambridge University Press)

Shih C F 1981 J. Mech. Phys. Solids 29305

Srinivas M, Malakondiah G and Ramarao P 1994a Proc. $R$. Soc. London A447 223

Srinivas M, Sundararajan G, Malakondiah $G$ and Ramarao P 1994b Proc. R. Soc. London A447 237

Sundararajan G 1993 Wear 162-164 773

Suresh K V, Ramakrishnan N, Srinivas M and Ramarao P 1999 J. Testing \& Evaluation (in press) 